Short Communication

Open Access

\title{
Stereochemistry of Consabatine from Convolvulus sabatius VIv. (Convolvulaceae)
}

\author{
Sonja Christina OTt, Kristina JeNETt-SIEMS, Eckart EICH *
}

Institut für Pharmazie (Pharmazeutische Biologie), Freie Universität Berlin, Königin-Luise-Str. 2-4, D-14195 Berlin, Germany.

* Corresponding author. E-mails: kjsiems@zedat.fu-berlin.de (K. Jenett-Siems), eckeich@zedat.fu-berlin.de (E. Eich)

Sci Pharm. 2013; 81: 247-250

doi:10.3797/scipharm.1208-14

Published: $\quad$ February $5^{\text {th }} 2013$

Accepted: $\quad$ February $5^{\text {th }} 2013$

Received: $\quad$ August $26^{\text {th }} 2012$

This article is available from: http://dx.doi.org/10.3797/scipharm.1208-14

(C) Ott et al.; licensee Österreichische Apotheker-Verlagsgesellschaft m. b. H., Vienna, Austria.

This is an Open Access article distributed under the terms of the Creative Commons Attribution License (http://creativecommons.org/licenses/by/3.0/), which permits unrestricted use, distribution, and reproduction in any medium, provided the original work is properly cited.

\begin{abstract}
The stereochemistry of consabatine, which was isolated from the roots of Convolvulus sabatius VIV. as a novel natural compound, has now been determined by the synthesis of its Mosher esters. Consabatine was found to be 1'R-configurated.
\end{abstract}

\section{Keywords}

Convolvulus sabatius $•$ Convolvulaceae $・$ Consabatine $\bullet 3 \alpha$-Tropanol ester $\bullet$ Mosher esters

\section{Introduction}

Tropane alkaloids are one of the most important and widespread groups of secondary metabolites in the Convolvulaceae. From the roots of the Mediterranean Convolvulus sabatius VıV., consabatine was isolated as a new natural compound [1]. This extraordinary $3 \alpha$-tropanol ester comprises an unusual isoprenylated cyclohexenylic acid as its acylic component. Mainly from the Merremia species, several related aromatic substances called merresectines - esters of 3a-tropanol with kurameric acid/nervogenic acid and derivatives - have been isolated as well [2]. Especially from the chemotaxonomic point of view, the identification of consabatine and the related merresectines is of significance, as they are specific to Convolvulaceae so far.

\section{Results and Discussion}

To clearly define a natural compound, the knowledge of not only its molecular structure, but also of its stereochemistry is essential. In order to determine the absolute configuration 
of C-1' in the terpenoid moiety of consabatine, the advanced Mosher method was applied [3]. After preparation of the epimeric Mosher esters, S-MTPA-consabatine and R-MTPAconsabatine, they were submitted for ${ }^{1} \mathrm{H}-\mathrm{NMR}$ spectroscopy. As described in [4], the differences in the protons' chemical shifts $\Delta \delta(S-R)$ between S-MTPA- and R-MTPAconsabatine were calculated (Fig. 1). According to [3], negative $\Delta \delta$-values point to an orientation above the MTPA plane (L3), and positive values to an orientation below the MTPA plane (L2). As a consequence, consabatine shows a 1'R-configuration.

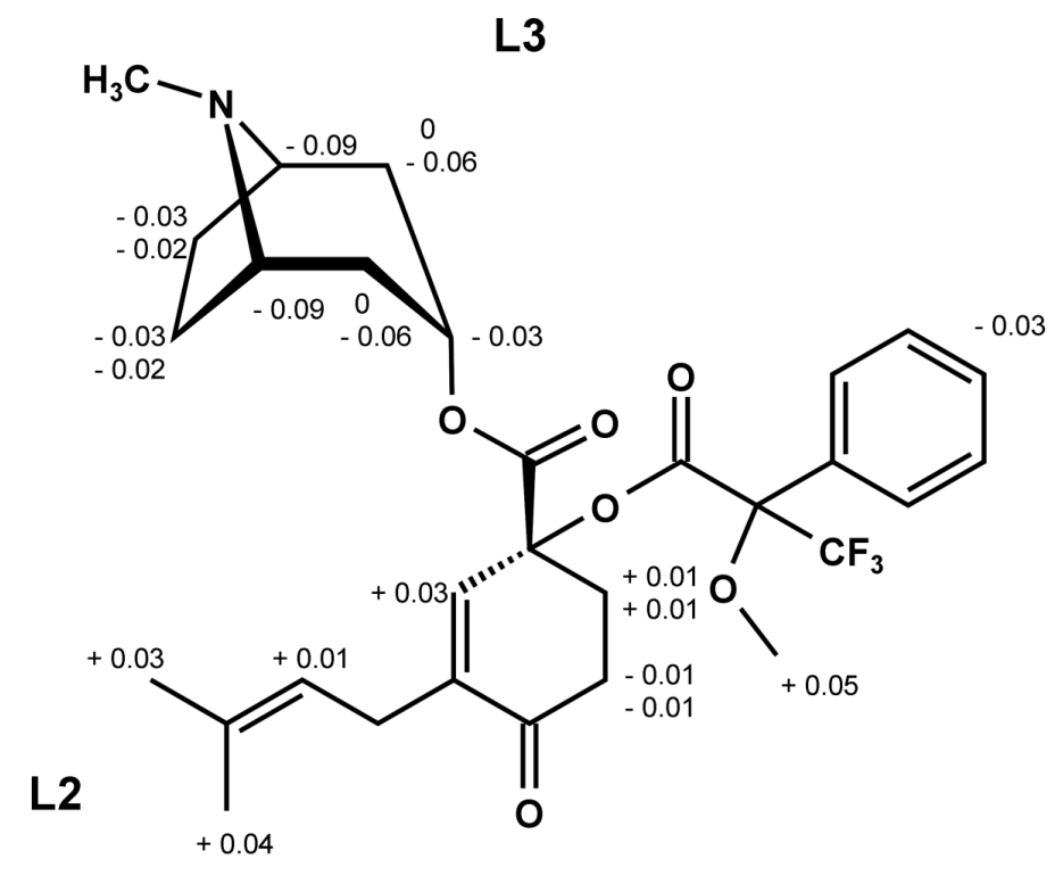

Fig. 1. MTPA-consabatine with differences $\Delta \delta(S-R)$ taken from the ${ }^{1} \mathrm{H}-\mathrm{NMR}$ spectra of S-MTPA- and R-MTPA-consabatine

\section{Experimental}

\section{General procedures}

${ }^{1} \mathrm{H}-\mathrm{NMR}$ and ${ }^{1} \mathrm{H}-{ }^{1} \mathrm{H}$-COSY spectra were obtained on a Bruker AMX $400 \mathrm{MHz}$ (TMS as internal standard). The EIMS was recorded on a Varian MAT 711 (70 eV).

\section{Plant material}

Several specimen of Convolvulus sabatius VIV. were bought at Gartencenter Pluta, Berlin. They were cultivated and harvested at the Berlin Botanical Garden.

\section{Extraction and isolation of consabatine}

The dried and ground roots of Convolvulus sabatius were extracted with methanol three times. After evaporation of the solvent, the residue was dissolved in $2 \%$ aqueous tartaric acid and extracted with petrolether, dichloromethane, and ethyl acetate. Then, the aqueous layer was alkalinized ( $\mathrm{pH}$ 10) with 25\% aqueous $\mathrm{NH}_{3}$ and extracted with dichloromethane again. This alkaloidal extract was separated by means of preparative HPLC (0.5\% aqueous $\mathrm{H}_{3} \mathrm{PO}_{4} / \mathrm{MeOH} 80: 20$ to $40: 60$ in $60 \mathrm{~min}$ ) and preparative TLC 
( $\mathrm{CHCl}_{3} / \mathrm{MeOH}$ /aq. $\mathrm{NH}_{3}$ Conc. 80:20:2). Consabatine was verified by ${ }^{1} \mathrm{H}-\mathrm{NMR}$ and EIMS measurements.

Consabatine (12.2 mg), (1R,3r,5S)-8-Methyl-8-azabicyclo[3.2.1]octan-3-yl (1R)-1-hydroxy3-(3-methylbut-2-en-1-yl)-4-oxocyclohex-2-ene-1-carboxylate:

${ }^{1} \mathrm{H}-\mathrm{NMR}\left(400 \mathrm{MHz}_{\mathrm{CDCl}}\right.$ ): $\delta 6.40(1 \mathrm{H}, \mathrm{s}, \mathrm{H}-2$ '), $5.10(2 \mathrm{H}, \mathrm{t}, \mathrm{J}=5.0 \mathrm{~Hz}, \mathrm{H}-3 / \mathrm{H}-2$ "), 3.14 (2H, br s, H-1/H-5), $2.96\left(2 \mathrm{H}\right.$, br d, J = $7.0 \mathrm{~Hz}, \mathrm{CH}_{2}-1$ "), $2.74(1 \mathrm{H}$, ddd, $J=5.5 \mathrm{~Hz}, 7.5 \mathrm{~Hz}$, and $\left.17.1 \mathrm{~Hz}, \mathrm{H}-5^{\prime} d\right), 2.62(1 \mathrm{H}$, ddd, J = 5.2 Hz, 8.9 Hz, and $17.1 \mathrm{~Hz}, \mathrm{H}-5$ 'u), $2.37(1 \mathrm{H}, \mathrm{tt}, \mathrm{J}$ $=5.6 \mathrm{~Hz}$, and $7.5 \mathrm{~Hz}, \mathrm{H}-6 \mathrm{~d}), 2.30\left(3 \mathrm{H}, \mathrm{s}, \mathrm{N}-\mathrm{CH}_{3}\right), 2.24(1 \mathrm{H}, \mathrm{dt}, \mathrm{J}=5.3 \mathrm{~Hz}$, and $8.7 \mathrm{~Hz}, \mathrm{H}-$ 6'u), 2.20 (2H, m, H-2ax/H-4ax), 2.02 (2H, m, H-6exo/H-7exo), 1.73 (3H, s, $\left.\mathrm{CH}_{3}-4 "\right), 1.72$

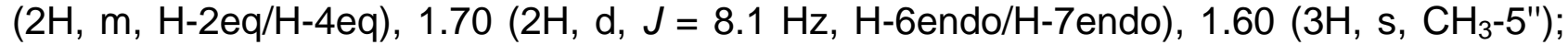
EIMS (70 eV): m/z (rel. int.) 347 (16), 330 (1), 223 (1), 141 (7), 140 (3), 125 (12), 124 (100), 97 (9), 96 (18), 95 (8), 94 (8), 83 (26), 82 (21).

\section{Synthesis of the Mosher esters of consabatine}

One-half of the consabatine obtained $(6.1 \mathrm{mg})$ was dissolved in $0.5 \mathrm{~mL}$ anhydrous dichloromethane. Then $8.8 \mathrm{mg}$ dimethylaminopyridine (DMAP), 3.7 $\mu \mathrm{L}$ triethylamine (TEA), and $6.6 \mu \mathrm{L}(-)$-a-methoxy- $\alpha$-(trifluoromethyl)phenylacetic acid (MTPA) chloride were added under nitrogen atmosphere. The mixture was stirred overnight. To terminate the reaction, $4.34 \mu \mathrm{L}$ 3-[(dimethylamino)propyl]amine (3-DMAPA) was added, and the mixture was stirred for $10 \mathrm{~min}$. After evaporation of the solvent, the residue was applied to the preparative TLC $\left(\mathrm{CHCl}_{3} / \mathrm{MeOH} / \mathrm{aq}\right.$. $\mathrm{NH}_{3}$ conc. 40:10:1) to give S-MTPA-consabatine.

S-MTPA-consabatine (4.2 mg): ${ }^{1} \mathrm{H}-\mathrm{NMR}\left(400 \mathrm{MHz}, \mathrm{CDCl}_{3}\right): \delta 7.36-7.45(5 \mathrm{H}, \mathrm{m}$, aromatic protons), $6.92\left(1 \mathrm{H}, \mathrm{s}, \mathrm{H}-2^{\prime}\right), 5.22(1 \mathrm{H}, \mathrm{t}, \mathrm{J}=4.4 \mathrm{~Hz}, \mathrm{H}-3), 5.06\left(1 \mathrm{H}, \mathrm{br} \mathrm{t}, \mathrm{J}=7.0 \mathrm{~Hz}, \mathrm{H}-2^{\prime \prime}\right)$, $3.78\left(1 \mathrm{H}, \mathrm{m}, \mathrm{H}-5^{\prime} \mathrm{d}\right), 3.76\left(1 \mathrm{H}, \mathrm{m}, \mathrm{H}-5{ }^{\prime} \mathrm{u}\right), 3.57\left(3 \mathrm{H}, \mathrm{s}, \mathrm{N}-\mathrm{CH}_{3}\right), 3.55\left(3 \mathrm{H}, \mathrm{s}, \mathrm{O}-\mathrm{CH}_{3}\right), 3.39$ $(2 \mathrm{H}, \mathrm{d}, \mathrm{J}=7.3 \mathrm{~Hz}, \mathrm{H}-2 \mathrm{ax} / \mathrm{H}-4 \mathrm{ax}), 3.09(2 \mathrm{H}, \mathrm{m}, \mathrm{H}-1 / \mathrm{H}-5), 2.95\left(2 \mathrm{H}, \mathrm{br} d, J=7.4 \mathrm{~Hz}, \mathrm{CH}_{2^{-}}\right.$ 1"), $2.47(2 \mathrm{H}, \mathrm{m}, \mathrm{H}-6 \mathrm{exo} / \mathrm{H}-7 \mathrm{exo}), 2.29(1 \mathrm{H}, \mathrm{m}, \mathrm{H}-6 \mathrm{~d}), 2.24(1 \mathrm{H}, \mathrm{m}, \mathrm{H}-6 \mathrm{u}), 2.12(2 \mathrm{H}$, br d, $\mathrm{J}=16.1 \mathrm{~Hz}, \mathrm{H}-6$ endo/H-7endo), 1.92 (2H, br d, $\mathrm{J}=16.6 \mathrm{~Hz}, \mathrm{H}-2 \mathrm{eq} / \mathrm{H}-4 \mathrm{eq}), 1.76$ (3H, s, $\left.\mathrm{CH}_{3}-4 "\right), 1.61\left(3 \mathrm{H}, \mathrm{s}, \mathrm{CH}_{3}-5 "\right)$.

The second half of consabatine (6.1 mg likewise) was treated in the same manner with (+)-MTPA chloride instead to give R-MTPA-consabatine.

R-MTPA-consabatine (3.7 mg): ${ }^{1} \mathrm{H}-\mathrm{NMR}\left(400 \mathrm{MHz}, \mathrm{CDCl}_{3}\right): \delta 7.38-7.49(5 \mathrm{H}, \mathrm{m}$, aromatic protons), $6.89\left(1 \mathrm{H}, \mathrm{s}, \mathrm{H}-2\right.$ ') $5.25(1 \mathrm{H}, \mathrm{t}, \mathrm{J}=5.1 \mathrm{~Hz}, \mathrm{H}-3), 5.05\left(1 \mathrm{H}, \mathrm{br} \mathrm{t}, \mathrm{J}=7.3 \mathrm{~Hz}, \mathrm{H}-2^{\prime \prime}\right)$, $3.78\left(1 \mathrm{H}, \mathrm{m}, \mathrm{H}-5^{\prime} \mathrm{d}\right), 3.75\left(1 \mathrm{H}, \mathrm{m}, \mathrm{H}-5\right.$ 'u), $3.54\left(3 \mathrm{H}, \mathrm{s}, \mathrm{N}-\mathrm{CH}_{3}\right), 3.50\left(3 \mathrm{H}, \mathrm{s}, \mathrm{O}-\mathrm{CH}_{3}\right), 3.39$ $(2 \mathrm{H}, \mathrm{d}, \mathrm{J}=7.2 \mathrm{~Hz}, \mathrm{H}-2 \mathrm{ax} / \mathrm{H}-4 \mathrm{ax}), 3.18(2 \mathrm{H}, \mathrm{m}, \mathrm{H}-1 / \mathrm{H}-5), 2.96\left(2 \mathrm{H}, \mathrm{br} d, J=7.0 \mathrm{~Hz}, \mathrm{CH}_{2^{-}}\right.$ 1"), $2.50(2 \mathrm{H}, \mathrm{m}, \mathrm{H}-6 \mathrm{exo} / \mathrm{H}-7 \mathrm{exo}), 2.30(1 \mathrm{H}, \mathrm{m}, \mathrm{H}-6 \mathrm{~d}), 2.25\left(1 \mathrm{H}, \mathrm{m}, \mathrm{H}-6{ }^{\prime} \mathrm{u}\right), 2.14(2 \mathrm{H}$, br d, $\mathrm{J}=15.8 \mathrm{~Hz}, \mathrm{H}-6$ endo/H-7endo), $1.98(2 \mathrm{H}, \mathrm{br} \mathrm{d}, \mathrm{J}=16.4 \mathrm{~Hz}, \mathrm{H}-2 \mathrm{eq} / \mathrm{H}-4 \mathrm{eq}), 1.73$ (3H, s, $\left.\mathrm{CH}_{3}-4 "\right), 1.57\left(3 \mathrm{H}, \mathrm{s}, \mathrm{CH}_{3}-5 "\right)$.

\section{Acknowledgement}

The authors are indebted to Ms. H. Wilke and Mr. M. Meyer (BGBM/FU Berlin) for the cultivation of Convolvulus sabatius and thank Ms. M. Meyer for a helping hand in the synthesis of the Mosher esters. 


\section{Authors' Statement}

\section{Competing Interests}

The authors declare no conflict of interest.

\section{References}

[1] Jenett-Siems K, Mann P, Kaloga M, Siems K, Jakupovic J, Witte L, Eich E.

Tropane alkaloids with a unique type of acyl moiety from two Convolvulus species.

Phytochemistry. 1998; 49: 1449-1451.

http://dx.doi.org/10.1016/S0031-9422(98)00083-1

[2] Jenett-Siems K, Weigl R, Böhm A, Mann P, Tofern-Reblin B, Ott S C, Ghomian A, Kaloga M, Siems K, Witte L, Hilker M, Müller F, Eich E.

Chemotaxonomy of the pantropical genus Merremia (Convolvulaceae) based on the distribution of tropane alkaloids.

Phytochemistry. 2005; 66: 1448-1464.

http://dx.doi.org/10.1016/j.phytochem.2005.04.027

[3] Ohtani I, Kusumi T, Kashman Y, Kakisawa H.

High-field FT NMR application of Moshers' method. The absolute configurations of marine terpenoids. J Am Chem Soc. 1991; 113: 4092-4096.

http://dx.doi.org/10.1021/ja00011a006

[4] Dale J A, Mosher H S.

Nuclear magnetic resonance enantiomer reagents. Configurational correlations via nuclear magnetic resonance chemical shifts of diastereomeric mandelate, O-methylmandelate, and $\alpha$-methoxya-trifluoromethylphenylacetate (MTPA) esters

J Am Chem Soc. 1973; 95: 512-519.

http://dx.doi.org/10.1021/ja00783a034 